\title{
Glaucoma surgery with or without adjunctive antiproliferatives in normal tension glaucoma: 1 Intraocular pressure control and complications
}

\author{
W L Membrey, D P Poinoosawmy, C Bunce, R A Hitchings
}

\begin{abstract}
Background-Reduction of intraocular pressure (IOP) by $20-30 \%$ with glaucoma drainage surgery slows disease progression in normal tension glaucoma (NTG). It is not clear whether adjunctive antiproliferative agents are necessary or safe in eyes at low risk for scarring.

Method-86 eyes of 73 white NTG patients who had undergone a primary guarded fistulising procedure were reviewed. 25 eyes had no antiproliferatives, 36 had peroperative 5-fluorouracil (5-FU) and 25 had peroperative mitomycin C (MMC). Their postoperative IOPs, complications, and changes in visual acuity were recorded.

Results-Eyes that had no adjunctive antiproliferative less commonly maintained a $20-30 \%$ reduction in IOP $(47.4 \%$ at 2 years) compared with either the $5-F U$ group $(69.4 \%$ at 2 years, $p=0.01)$ or the MMC group $(64.9 \%$ at 2 years, $p=0.04)$. Eyes that had adjunctive MMC more often had late hypotony $(28 \%, p=0.02)$ and late bleb leak $(12 \%, p<0.001)$. Eyes that had adjunctive MMC also more often had a two lines loss of Snellen visual acuity $(39.8 \%$ by 2 years) compared with those that had adjunctive $5-F U(14.7 \%$ by 2 years), $p=0.06$.

Conclusion-For NTG patients at low risk of scarring trabeculectomy with adjunctive peroperative $5-\mathrm{FU}$ should maintain a suitable target IOP without the additional sight threatening complications seen with adjunctive MMC.

(Br f Ophthalmol 2000;84:586-590)
\end{abstract}

Normal tension glaucoma (NTG) has a prevalence of $0.6 \%$ within white populations and is thought to account for $20-30 \%$ of primary open angle glaucoma. ${ }^{1-3}$ It is characterised by an intraocular pressure (IOP) that is found to be within the normal range together with cupping of the optic nerve head and visual field loss of a nerve fibre layer type. Progressive visual field loss may be detected in $75-84 \%$ of NTG within 5 years of follow up. ${ }^{4}$

The treatment of progressive NTG represents a therapeutic challenge. The pathogenesis of this condition is thought to be multifactorial. IOP plays a part in the continuation of glaucomatous damage. ${ }^{5}$ This has been suggested by the observation that the eye with the higher pressure has the more severe disease ${ }^{67}$ and by interventions that reduce IOP and slow disease progression. ${ }^{89}$
Treatment for NTG has therefore concentrated on lowering IOP. Glaucoma surgery has been shown to slow the rate of progression of this disease. ${ }^{10-12}$ This beneficial effect occurs with IOP reductions of $25-30 \%$ from baseline. ${ }^{10}{ }^{13-16}$ Because of the risk of failure to achieve low normal IOP in the postoperative period guarded fistulising procedures with or without the use of adjunctive postoperative or perioperative 5 -fluorouracil ${ }^{9}$ or perioperative mitomycin $\mathrm{C}^{17}$ have been proposed.

Because target IOP is lower, NTG patients are at greater risk of ocular hypotony and associated complications such as hypotonous maculopathy ${ }^{18}$ and cataract. ${ }^{19}$ The collaborative NTG study found that the progression of cataract in the surgically treated patients confounded any beneficial effect on glaucomatous visual field progression. These additional risks are likely to be increased by the use of adjunctive antiproliferative therapy in these patients.

Because it is not clear whether antiproliferative agents are required to achieve target IOP in low risk NTG patients without exposing the patient to an unacceptable risk of potentially sight threatening complications we reviewed our patients who had undergone surgical treatment for progressive NTG with or without antiproliferative agents.

\section{Materials and methods}

Patients attending the normal tension glaucoma clinic at Moorfields Eye Hospital who had been treated with drainage surgery (trabeculectomy) at least 6 months before the time of data collection were included in the study. To be classified as NTG patients had to have: (1) glaucomatous cupping of the optic nerve head, (2) reproducible visual field loss of a retinal nerve fibre layer type on static automated perimetry, (3) untreated mean phasing IOP no greater than $21 \mathrm{~mm} \mathrm{Hg}$ and no single reading greater than $23 \mathrm{~mm} \mathrm{Hg}$. The decision to institute treatment had been taken when the patient demonstrated glaucomatous progression using pointwise linear regression analysis (PROGRESSOR $^{2021}$ ) of their automated Humphrey 24/2 visual field series.

Patients' hospital records were reviewed and the following data collected: sex; date of birth; race; date of surgery; use of adjunctive antiproliferative agent; type of conjunctival flap; use of glaucoma medications preoperatively and postoperatively; preoperative and postoperative IOP; complications following drainage surgery; history of other ocular surgery and changes in visual acuity. 
All non-white patients and patients with a history of previous surgery involving incision of the conjunctiva or penetration of the eye or whose drainage procedure was combined with cataract extraction were excluded.

All patients had undergone guarded fistulising procedure under a limbal or fornix based conjunctival flap. This was done either without the use of adjunctive antiproliferative therapy (designated the nil adjunct group), with the application of $25 \mathrm{mg} / \mathrm{ml} \mathrm{5-FU} \mathrm{for} 5$ minutes to the scleral bed before penetration of the eye (designated the 5-FU group) or with the application of mitomycin C $0.1 \mathrm{mg} / \mathrm{ml}$ for 3-5 minutes to the scleral bed before penetration of the eye (designated the MMC group). The use or otherwise of peroperative antiproliferative agents followed an evolving policy in the NTG clinic. The first series did not receive any, the second received 5-FU, and the third MMC. The operations in all groups were performed by surgeons with varied experience including consultants, fellows, and residents in training at the glaucoma unit in Moorfields Eye Hospital.

The patients were seen on a routine basis twice yearly, with more frequent visits during the postoperative period, and as occasion required. If patients had drainage surgery to both eyes within the same adjunct group only the first eye to undergo drainage surgery was included in the analysis. This was done because the right and left eyes of an individual patient are known to be interdependent. The inclusion of both eyes within the same adjunct group might therefore introduce bias owing to the overrepresentation of that patients predisposition to failure or success within that group. However, we included both eyes when they fell into different adjunct groups as to do so would increase the power of the study to detect a difference in outcome between these groups.

The median of the IOPs in the 2 years before surgery for each patient was taken as the preoperative baseline. The median IOP and corresponding percentage IOP reduction from the baseline was recorded at 1 month, 3 months, and for each 6 month period postoperatively. Patients were classified as failing to achieve adequate IOP reduction if this was less than $25 \%$ from preoperative levels on two successive occasions from 3 months postoperatively. Time to IOP failure was taken to be the first of these occasions. Patients who did not satisfy the IOP failure criteria above but who nevertheless required repeat filtering surgery due to inadequate IOP reduction and/or continued progression were also classified as failing. Time to failure was defined in these patients as the time between the first and second operations. The above procedure was repeated using less than $20 \%$ and $30 \%$ IOP reduction as the definition of failure.

Visual acuity was recorded as the best of the corrected or pinhole vision during each 6 month follow up period.

Eyes that lost two or more Snellen lines of visual acuity or worsened by one low vision category (low vision categories were counting fingers, hand movement, light perception, and no light perception) consistently in two successive 6 month follow up periods from 3 months postoperatively were identified and recorded as "visual acuity failures". The time to visual acuity drop was recorded as the time from drainage surgery until the first of these.

Complications were divided into early and late. Early complications were those occurring in the perioperative or early postoperative period. Late complications were after the initial healing phase had been completed, and were considered to be those seen 3 months or more after surgery. Perioperative or early postoperative complications included: anterior chamber hyphaema; shallowing of the anterior chamber; choroidal effusions; early hypotony (which was defined as an IOP less than or equal to $5 \mathrm{~mm}$ $\mathrm{Hg}$ on two successive occasions at least 4 weeks apart); and early bleb leak.

Late postoperative or sight threatening complications included: bleb leak; infective "blebitis" or endophthalmitis; hypotonous maculopathy; and late hypotony. The number of eyes in each group undergoing cataract surgery and the time from drainage surgery to cataract surgery was also noted.

"IOP failure", "visual acuity failure", and cataract surgery were analysed using survival analysis techniques. Kaplan-Meier plots were constructed and Cox regression performed. Log rank tests were conducted to assess evidence of differences in "survival" experience following use of adjunctive antiproliferative or no adjunctive antiproliferative. Categorical outcomes were compared between the three groups using $\chi^{2}$ tests or Fisher's exact test where appropriate. Baseline quantitative data were compared using the Kruskal-Wallis procedure. Analyses were conducted with sPSS version 8.0 for windows and Stata Statistical Software Release 5 (Stata Corporation, USA).

\section{Results}

One hundred and six eyes of 86 patients had drainage surgery 6 or more months before data collection. Six eyes of six patients were excluded because of previous surgery or combined surgery. Six eyes of six patients were non-white and were therefore excluded. One eye of one patient was excluded because the hospital records could not be found. Seven patients had the same type of adjunct to both eyes, the second eye being excluded from all analysis.

This left 86 eyes (49 left and 37 right) of 73 patients (52 females and 21 males).

USE OF ADJUNCTIVE ANTIPROLIFERATIVE THERAPY There were 25 eyes $(29.1 \%)$ in the nil adjunct group, 36 eyes $(41.8 \%)$ in the 5 -FU group, and 25 eyes $(29.1 \%)$ in the MMC group. Drainage surgery was performed between May 1986 and July 1992 for the majority of patients in the nil adjunct group (four of 25 patients had surgery after this and therefore overlapped with the other two groups), between November 1992 and September 1995 in the majority of patients in the 5-FU group (one patient overlapping with the MMC group) and between September 1995 and March 1998 in the MMC group. 
Table 1 Preoperative and postoperative quantitative data and postoperative categorical data excluding complications. Medians were compared using the Kruskal-Wallis procedure, categorical data was compared using $\chi^{2}$ test or Fisher's exact test where appropriate

\begin{tabular}{|c|c|c|c|c|}
\hline & Nil adjunct group & 5-FU group & $M M C$ group & $p$ Value $(2 d f)$ \\
\hline Median age at surgery (IQ range) & $61.5(51.4-67.7)$ & $67.7(61.4-74)$ & $68.4(60.2-72.6)$ & 0.019 \\
\hline Median preoperative follow up (IQ range) & $55.1(20.1-94.3)$ & $80.3(31.4-136.4)$ & $96.1(52.3-126.4)$ & 0.04 \\
\hline Median postoperative follow up (IQ range) & $96(60-126)$ & $45(35.5-54)$ & $18(12-18)$ & 0.0001 \\
\hline Median of 3 MDs closest to date of surgery (IQ range) & $-13.6(-22.5$ to -11$)$ & $-16.7(-19.9$ to -11.9$)$ & $-12.6(-18.2$ to -10.2$)$ & 0.286 \\
\hline Number of left eyes & $17 / 25(68 \%)$ & $20 / 36(55.6 \%)$ & $12 / 25(48 \%)$ & 0.352 \\
\hline Female & $19 / 25(76 \%)$ & $28 / 36(77.8 \%)$ & $15 / 25(60 \%)$ & 0.275 \\
\hline Preoperative drops & $16 / 24(66.7 \%)$ & $24 / 34(70.6 \%)$ & $11 / 25(44 \%)$ & 0.096 \\
\hline Preoperative drops in year before surgery & $8 / 24(33.3 \%)$ & $14 / 34(42.2 \%)$ & $7 / 25(28 \%)$ & 0.566 \\
\hline Postoperative IOP lowering dops & $7 / 25(28 \%)$ & $5 / 36(14.3 \%)$ & $1 / 25(4 \%)$ & 0.06 \\
\hline Redo trabeculectomy & $3 / 25(12 \%)$ & $2 / 36(5.6 \%)$ & $1 / 25(4 \%)$ & 0.49 \\
\hline
\end{tabular}

Details on the type of conjunctival incision were not provided for patients in either the nil adjunct group or the 5-FU group. The standard drainage surgery for these groups during the study period was a trabeculectomy with a fornix based conjunctival and scleral flap. For the MMC group there were 23 patients where the type of conjunctival flap was recorded and 20 of these were limbus based. A subanalysis based on the type of conjunctival flap is not possible with these small numbers.

Demographic data including median age and median mean deviations (MDs) and preoperative and postoperative follow up are shown (see Table 1).

\section{PREOPERATIVE TOPICAL MEDICATION}

There were incomplete records on three eyes from three patients (one from the nil adjunct group and two from the 5-FU group); these eyes were left out of this part of the analysis.

There was no significant difference (at the $\mathrm{p}<0.05$ level) between the three groups in the proportion of subjects who were taking topical IOP lowering medications at any time preoperatively or in the year leading up to drainage surgery (see Table 1$)$. Six $(24.0 \%)$ of the nil adjunct group had only been on topical $\beta$ blockers and $10(40 \%)$ had been taking adrenaline and/or pilocarpine at some time before drainage surgery. These figures were 11 $(30.5 \%)$ and $12(33.3 \%)$ respectively for the 5 -FU group and eight $(32.0 \%)$ and three $(12 \%)$ respectively for the MMC group.

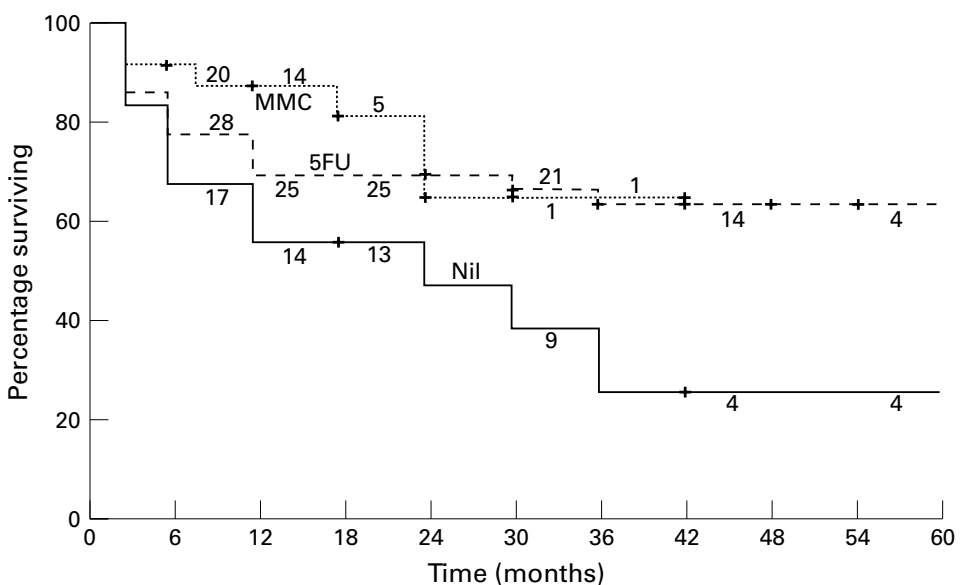

Figure 1 Failure as defined by less than 25\% drop in IOP from the time of surgery or redo drainage surgery by adjunct group.
IOP

Comparison of the three groups using the log rank test showed a statistically significant increase in survival time in both the 5-FU and MMC groups compared with the nil adjunct group. The difference between the 5-FU group and the MMC group did not reach statistical significance (see Fig 1). Thirty eight patients failed to maintain $25 \%$ IOP reduction as defined above. Five of these occurred in the MMC group, 13 in the 5-FU group, and 20 in the nil adjunct group. The cumulative failures at 1 year and 2 years was $44 \%$ and $52.6 \%$ for the nil adjunct group, $30.6 \%$ and $30.6 \%$ for the $5-\mathrm{FU}$ group, and $12.6 \%$ and $35.1 \%$ for the MMC group. The log rank statistic between the nil adjunct and 5-FU groups was 6.6 $(\mathrm{p}=0.0102)$, between nil and MMC groups was $4.04(p=0.0445)$, and between 5-FU and MMC groups was $0.58(\mathrm{p}=0.4468)$.

Cox regression analysis showed no statistically significant effect of sex, age, or preoperative drop use on "IOP survival" when adjusted for adjunct group.

POSTOPERATIVE IOP REDUCING MEDICATIONS AND REPEAT DRAINAGE SURGERY

Thirteen eyes were started on topical IOP reducing medication postoperatively. Two of these continued to maintain a $25 \%$ reduction in IOP, one eye from the 5-FU group and one from the MMC group. Six eyes, four from the nil adjunct group, one from the 5-FU group, and one from the MMC group had further glaucoma drainage surgery (see Table 1 ).

POSTOPERATIVE VISUAL ACUITY

Two eyes in the 5-FU group had visual acuity loss attributed to other causes, one to macular epiretinal membrane formation postoperatively and one to age related macular degeneration. These eyes have been left out of the analysis.

Kaplan-Meier survival analysis using the above definition of "visual acuity failure" showed a poorer survival in the MMC group compared with the 5-FU group, albeit one that did not quite achieve the usually accepted level of statistical significance $(p=0.06)$. The differences between the nil adjunct group and both the 5-FU group and the MMC group did not reach statistical significance (see Fig 2). There were 26 "visual acuity failures", nine of which occurred in the MMC group, eight in the 5-FU group, and nine in the nil adjunct group. The proportion of patients losing two or more lines of Snellen visual acuity at 1 and 2 years was 


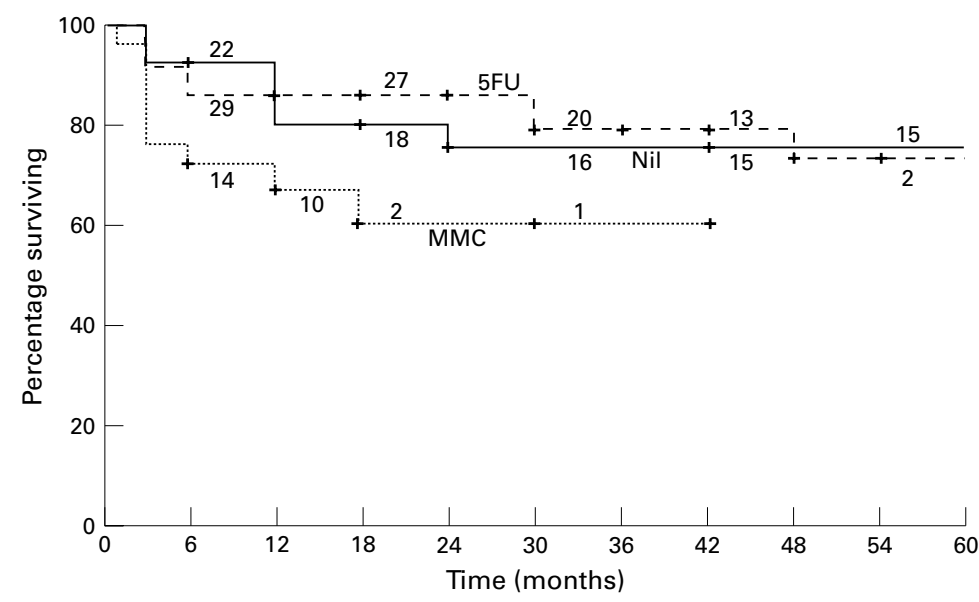

Figure 2 Failure as defined by a two line drop in Snellen visual acuity from the time of surgery by adjunct group.

$20.6 \%$ and $25.0 \%$ for the nil adjunct group, $14.7 \%$ and $14.7 \%$ for the 5 -FU group, and $33.1 \%$ and $39.8 \%$ for the MMC group. The log rank statistic between nil and 5-FU groups was $0.0(p=0.9668)$, between nil and MMC groups was $2.13(\mathrm{p}=0.1441)$, and between 5-FU and MMC groups was $3.84(\mathrm{p}=0.06)$.

\section{PERIOPERATIVE AND EARLY POSTOPERATIVE}

COMPLICATIONS

Early postoperative complications are shown in Table 2. There was no significant difference in the frequency of these early postoperative complications between the three adjunct groups (Pearson's $\chi^{2}$ statistic $\mathrm{p}>0.05$ ).

\section{LATE POSTOPERATIVE OR SIGHT THREATENING COMPLICATIONS}

The late and sight threatening complications are set out in Table 2. It will be seen that there was a significantly greater proportion of eyes in the MMC group with hypotony and bleb leaks.

\section{Discussion}

At the present time IOP reduction is the only form of treatment for NTG that has been shown to have any long term effect on the course of the disease. ${ }^{812}$ As the reduction needed has to be $20 \%$ or more ${ }^{1013-15}$ fistulising surgery is likely to have a major role in achieving this end, although some studies have reported IOP reductions in this range with topical therapy and laser trabeculoplasty alone. $^{22}$ The purpose of this paper was to

Table 2 Perioperative and early postoperative complications and late or sight threatening complications. Data were compared using $\chi^{2}$ test or Fisher's exact test where appropriate

\begin{tabular}{lllll}
\hline & $\begin{array}{l}\text { Nil adjunct } \\
\text { group }\end{array}$ & 5-FU group & MMC group & $\begin{array}{c}\text { p Value } \\
(2 d f)\end{array}$ \\
\hline $\begin{array}{l}\text { Perioperative or early complications } \\
\text { Any operative complications }\end{array}$ & $11 / 25(44 \%)$ & $19 / 36(52.8 \%)$ & $18 / 25(72 \%)$ & 0.122 \\
Hyphaea & $2 / 25(8 \%)$ & $6 / 36(16.7 \%)$ & $5 / 25(20 \%)$ & 0.48 \\
Shallow AC & $5 / 25(20 \%)$ & $4 / 36(11.1 \%)$ & $4 / 25(16 \%)$ & 0.644 \\
Early hypotony & $7 / 25(28 \%)$ & $9 / 36(25 \%)$ & $12 / 25(48 \%)$ & 0.143 \\
Early leaking bleb & $4 / 25(16 \%)$ & $7 / 36(19.4 \%)$ & $2 / 25(8 \%)$ & 0.475 \\
Choroidal effusion & $3 / 25(25 \%)$ & $7 / 36(19.4 \%)$ & $4 / 25(16 \%)$ & 0.82 \\
Late or sight threatening complications & & & & \\
Late bleb leak & $0 / 25(0 \%)$ & $0 / 36(0 \%)$ & $3 / 25(12 \%)$ & 0.045 \\
Late hypotony & $0 / 25(0 \%)$ & $0 / 36(0 \%)$ & $7 / 25(28 \%)$ & 0.0001 \\
Cataract surgery & $2 / 25(8 \%)$ & $3 / 36(8.3 \%)$ & $3 / 25(12 \%)$ & 0.9 \\
Endophthalmitis & $0 / 25(0 \%)$ & $0 / 36(0 \%)$ & $2 / 25(8 \%)$ & 0.164 \\
Hypotonous maculopathy & $1 / 25(4 \%)$ & $1 / 36(2.8 \%)$ & $3 / 25(12 \%)$ & 0.331 \\
\hline
\end{tabular}

review the results of fistulising surgery in its ability to achieve and maintain a target IOP reduction and to see what complications occur. We have looked at the results for three groups of patients, divided according to the use or otherwise of peroperative antiproliferative agents. The population studied was a "low risk" of failure group ${ }^{23}$ justifying no adjunctive therapy at the outset. The decision to use peroperative antiproliferatives was largely evolutionary, rather than based on case selection, so that a reasonable comparison can be made between the three groups when assessing the long term results. There was no significant difference between the three groups in preoperative IOP, use of preoperative glaucoma medication, and level of visual field loss as indicated by mean deviation on automated perimetry at the time of surgery.

The definition of "success" was entirely numeric, and did not imply an outcome in terms of visual field survival. The effect of such a lowering in IOP is to be addressed in a companion paper (Membrey et al, in preparation). Similarly the end point for "failure" was also an arbitrary one and based solely on IOP. Failure to maintain a target IOP reduction does not imply that the patient should undergo immediate reoperation or even introduction of medical therapy (although in practice this is common).

Our patients showed that peroperative 5-FU administered as described provided a significant improvement in success $(69.4 \%$ at 2 years) compared with the nil adjunct group ( $47.4 \%$ at 2 years), without the long term complications seen in the MMC group. Other patient groups, perhaps with more risk factors for failure, or a different method of administering MMC, such as with a fornix based conjunctival flap, might not have the long term complications, while maintaining the IOP survival rates. In a previous prospective study trabeculectomy with a limbus based conjunctival flap has been shown to produce similar IOP reduction to fornix based flaps with a tendency towards more short term complications only. ${ }^{25}$ However this was in high pressure glaucoma with a much shorter follow up (mean 11 months).

Apart from the long term complications noted above the effect of the IOP reduction on visual acuity needs to be recorded. By 18 months, $15-20 \%$ in the nil adjunct and 5-FU and $40 \%$ in the MMC groups had dropped two lines of Snellen visual acuity. This means that the best recorded Snellen vision whether with correction or pinhole from two successive 6 month period was at least two lines worse than the preoperative level. This loss of visual acuity may have occurred for a variety of reasons. Loss of central field and visual acuity due to glaucoma progression has been reported to occur postoperatively in advanced glaucoma, although it is an uncommon event. ${ }^{26}$ Progression of lens opacities and hypotonous maculopathy are more likely causes of visual acuity loss after drainage surgery. ${ }^{27}{ }^{28}$ Although not assessed in this study lens opacification after drainage surgery is more common in older patients ${ }^{28}$ and with postoperative 
hypotony, ${ }^{19}$ both of which are more likely in NTG patients. ${ }^{29}$ Although cataract extraction in patients who have lost vision due to lens opacification has the potential to restore sight it should be weighed against potential complications which include failure of the original drainage surgery. ${ }^{30} 31$

The greater loss of visual acuity in the MMC group may be accounted for by either clinically undetected hypotonous maculopathy or lens opacity progression. Both of these may have been more likely in this group owing to the more frequent occurrence of late postoperative hypotony. Other causes of loss of visual acuity might be refractive changes and ocular surface changes induced by the presence of a drainage bleb. Although it is difficult to know what was the cause of this visual loss, it does represent functionally significant morbidity.

This paper does not address outcomes in terms of visual field survival. In the light of changes in visual acuity noted here, surgery needs to be justified. It should be noted that all patients undergoing surgery in this series had documented visual field progression. A long term analysis of the incidence of progression for patients in our clinic has shown that 16-25\% will not have documented progression over 5 or more years of follow up. ${ }^{4}$ Clearly, surgery would not have been justified in this subset. Many of the patients with NTG are elderly and would be at risk of developing lens opacities. These opacities would enhance the visual field defects and mask any progression from their glaucoma, ${ }^{10}$ as well as reducing central acuity. Glaucoma surgery would not be helpful in this group. Many patients have slowly progressing disease, and surgery may not be needed for this group. Surgery remains a good therapeutic option for the patient with disease progression at a rate likely to cause significant functional loss of vision in their lifetime. For patients similar to the cohort presented here, a trabeculectomy with adjunctive 5-FU might be expected to achieve and maintain a suitable target IOP in the majority of cases.

It should be noted that the results presented here come from a retrospective analysis of data. Although we have looked for the effect of potential confounding factors, unexpected confounding factors cannot be excluded which might provide an alternative explanation for the data. Only a prospective randomised trial can provide definitive evidence, although these results should be useful in guiding the clinical management of these patients until this evidence becomes available.

This project was supported by the International Glaucoma Association.

1 Bonomi L, Marchini G, Marraffa M, et al. Prevalence of glaucoma and intraocular pressure distribution in a defined population. The Egna-Neumarkt Study. Ophthalmology 1998;105:209-15

2 Coffey M, Reidy A, Wormald R, et al. Prevalence of glaucoma in the west of Ireland. Br f Ophthalmol 1993;77: glaucoma $17-21$.

3 Dielemans I, Vingerling J, Wolfs R, et al. The prevalence of primary open-angle glaucoma in a population-based study in the Netherlands. The Rotterdam Study. Ophthalmology 994;101:1851-5.

4 Membrey WL, Poinoosawmy DP, Bunce C, et al. In normal tension glaucoma, eyes with visual field loss threatening fixation have no greater risk of continued visual field progression than eyes without threat to fixation. Invest Ophthalmol Vis Sci 1999;40:S65.

5 Araie M, Sekine M, Suzuki Y, et al. Factors contributing to the progression of visual-field damage in eyes with normaltension glaucoma. Ophthalmology 1994;101:1440-4.

6 Choplin NT. Correlation of asymmetric damage with asymmetric intraocular-pressure in normal-tension glaucoma (low-tension glaucoma). Arch Ophthalmol 1989;107:167-8.

7 Cartwright MJ, Anderson DR. Correlation of asymmetric damage with asymmetric intraocular-pressure in normaltension glaucoma (low-tension glaucoma). Arch Ophthalmol 1988;106:898-900.

8 Abedin S, Simmons RJ, Grant WM. Progressive low-tension glaucoma: treatment to stop glaucomatous cupping and field loss when these progress despite normal intraocular pressure. Ophthalmology 1982;89:1-6.

9 Wilson RP, Steinmann WC. Use of trabeculectomy with postoperative 5 -fluorouracil in patients requiring extremely ow intraocular pressure levels to limit further glaucoma progression. Ophthalmology 1991;98:1047-52.

10 Collaborative Normal-Tension Glaucoma Study Group. Comparison of glaucomatous progression between untreated patients with normal-tension glaucoma and patients with therapeutically reduced intraocular pressures [see?. Am f Ophthalmol 1998;126:487-97.

11 Bhandari A, Crabb DP, Poinoosawmy D, et al. Effect of surgery on visual field progression in normal-tension glaucoma. Ophthalmology 1997;104:1131-7.

12 Koseki N, Araie M, Shirato S, et al. Effect of trabeculectomy on visual field performance in central 30 degrees field in progressive normal-tension glaucoma. Ophthalmology 1997;104:197-201.

13 De Jong N, Greve EL, Hoyng PF, et al. Results of a filtering procedure in low tension glaucoma. Int Ophthalmol 1989;13:131-8.

14 Hitchings RA, Wu J, Poinoosawmy D, et al. Surgery for normal tension glaucoma [see comments]. Br f Ophthalmol 1995;79:402-6

15 Fontana L, Viswanathan AC, Poinooswamy D, et al. Surgery for normal tension glaucoma. Target intraocular pressure and visual field progression. Acta Ophthalmol Scand $1997 ; 75: 43-4$

16 Bhandari A, Crabb DP, Poinoosawmy D, et al. Effect of surgery on visual field progression in normal-tension glaucoma. Ophthalmology 1997;104:1131-7.

17 Yamamoto $\mathrm{T}$, Ichien $M$, Suemori-Matsushita $\mathrm{H}$, et al. [Trabeculectomy for normal-tension glaucoma]. Nippon Ganka Gakkai Zasshi-Acta Societatis Ophthalmologicae faponicae 1994;98:579-83.

18 Zacharia PT, Deppermann SR, Schuman JS. Ocular hypotony after trabeculectomy with mitomycin C. $A m$ f Ophthalmol 1993;116:314-26.

19 Vesti E. Development of cataract after trabeculectomy. Acta Ophthalmol Copenh 1993;71:777-81.

20 McNaught AI, Crabb DP, Fitzke FW, et al. Modelling series of visual fields to detect progression in normal-tension glaucoma. Graefes Archr Clin Exp Ophthalmol 1995;233: glaucoma

21 Viswanathan AC, Fitzke FW, Hitchings RA. Early detection of visual field progression in glaucoma: a comparison of PROGRESSOR and STATPAC 2. Br F Ophthalmol 1997;81: 1037-42.

22 Schulzer M. Intraocular pressure reduction in normaltension glaucoma patients. The Normal Tension Glaucoma Study Group. Ophthalmology 1992;99:1468-70.

23 Sturmer J, Broadway DC, Hitchings RA. Young patient trabeculectomy. Assessment of risk factors for failure. $O p h$ thalmology 1993;100:928-39.

24 Broadway D, Grierson I, Hitchings R. Racial differences in the results of glaucoma filtration surgery: are racial differences in the conjunctival cell profile important? $\mathrm{Br} F$ Ophthalmol 1994;78:466-75.

25 Grehn F, Mauthe S, Pfeiffer N. Limbus-based versus fornix-based conjunctival flap in filtering surgery. A randomized prospective study. Int Ophthalmol 1989;13: 139-43.

26 Henry JC. Snuff syndrome. F Glaucoma 1994;3:92-5.

27 Bayer AU, Erb C, Ferrari F, et al. The Tuebingen Glaucoma Study. Glaucoma filtering surgery-a retrospective longterm follow-up of 254 eyes with glaucoma. Ger $\mathcal{F}$ Ophthalmol 1995;4:289-93.

28 Costa VP, Smith M, Spaeth GL, et al. Loss of visual acuity after trabeculectomy. Ophthalmology 1993;100:599-612.

29 Zacharia PT, Deppermann SR, Schuman JS. Ocular hypotony after trabeculectomy with mitomycin C. Am f Ophthalmol 1993;116:314-26.

30 Seah SK, Jap A, Prata JA Jr, et al. Cataract surgery after trabeculectomy. Ophthalmic Surg Lasers 1996;27:587-94.

31 Chen PP, Weaver YK, Budenz DL, et al. Trabeculectomy function after cataract extraction. Ophthalmology 1998;105: 1928-35. 